Article

\title{
On the Equilibria of Generalized Dynamical Systems
}

\author{
Vasile Postolică \\ Department of Mathematics, Informatics and Training Sciences, Faculty of Sciences, "Vasile \\ Alecsandri” Bacău University, Calea Marasesti 157, 600115, Bacău, Romania; \\ E-Mail: vpostolica@ambra.ro; Tel.: +40-722-443-287; Fax: +40-233-218-197
}

Received: 24 September 2012; in revised form: 26 November 2012 / Accepted: 26 November 2012 /

Published: 6 December 2012

\begin{abstract}
This research work presents original properties of the equilibrium critical (ideal) points sets for an important class of generalized dynamical systems. The existence and significant results regarding such points are specified. Strong connections with the Vector Optimization by the Efficiency and the Potential Theory together with its applications following Choquet's boundaries are provided.
\end{abstract}

Keywords: Isac's cone; dynamical system; critical point; efficiency; Choquet's boundary

\section{Introduction}

In this research work we present properties of critical points for an important class of generalized dynamical systems through the connections with the efficiency and Choquet's boundaries. Section 2 is therefore devoted to the study of the existence and the properties of the critical point sets. In Section 3, two important coincidence results between the (approximate) critical point sets and the Choquet's boundaries are given. The last section contains a new approximate modality for this kind of equilibrium point sets. All the elements of ordered topological vector spaces used in this work are in accordance with [1].

\section{Existence of the Equilibrium Points under Completeness}

In this section we study the existence of the critical points for a class of generalized dynamical systems in separated locally convex spaces ordered by the (weak) supernormal cones introduced by G. Isac in [2] and published in [3], using the (weak) completeness. We note that the weakly complete cones are very important in the functional analysis [4,5] and in the potential theory, including their applications [6-10]. 
Let $X$ be a real Hausdorff locally convex space with the topology induced by a family $P=\left\{p_{\alpha}: \alpha \in I\right\}$ of seminorms, ordered by a convex cone $K$, its topological dual space $X^{*}$ and the origin denoted by $\theta$.

Definition 2.1 [3] $K$ is called an Isac's (nuclear or supernormal) cone if for every $p_{\alpha} \in P$ there exists $f \in X^{*}$ such that $p_{\alpha}(x) \leq f(x)$ for all $x \in K$.

Remark 2.1 We have named cones such as these "Isac's cone" in memory of my friend, Professor Isac as described in $[2,3,11]$. Many examples of such convex cones, their importance for efficiency and the last extension by the full nuclearity are described in [2,3,11-20] and elsewhere. Thus, in a normed linear space a convex cone is an Isac's cone iff it is well based, that is, generated by a non-empty, convex and bounded set which does not contain the origin in its closure. A convex cone is an Isac's cone in a nuclear space [21] if and only if it is a normal cone. Both these properties are not valid in general Hausdorff locally convex spaces. We also note that this concept is not a simple generalization of the corresponding notion defined in the normed linear spaces by M. A. Krasnoselski [22], being specific to the ordered Hausdorff locally convex spaces [23].

Let $A$ be a non-empty set in a Hausdorff locally convex space $X$ ordered by a convex cone $K$.

Definition 2.2 A set valued map $\Gamma: A \rightarrow 2^{A}$ is called a generalized dynamical system if $\Gamma(a)$ is non-empty for every $a \in A$.

Let $\Gamma: A \rightarrow 2^{A}$ be the generalized dynamical system defined by $\Gamma(a)=A \cap(a-K), a \in A$.

Definition 2.3 We say that $a_{0}$ is a critical point for $\Gamma$ or an efficient (minimum) point for $A$ with respect to $K$, in notation, $a_{0} \in \operatorname{MIN}_{K}(A)\left(a_{0} \in \operatorname{eff}(A, K)\right)$ if it satisfies one of the following equivalent conditions;

(i) $A \cap\left(a_{0}-K\right) \subseteq a_{0}+K$

(ii) $K \cap\left(a_{0}-A\right) \subseteq-K$

(iii) $(A+K) \cap\left(a_{0}-K\right) \subseteq a_{0}+K$

(iv) $K \cap\left(a_{0}-A-K\right) \subseteq-K$

It is clear that the finite dimensional version of this concept is represented by the Pareto optimality [20,24,25] and references therein. We recall that every Isac's cone is pointed, that is, $K \cap(-K)=\{\theta\}$ and in any such case as this $a_{0} \in \operatorname{MIN}_{K}(A)$, if and only if, $A \cap\left(a_{0}-K\right)=\left\{a_{0}\right\}$, or equivalently $K \cap\left(a_{0}-A\right)=\{\theta\}$. A projection in vector optimization by the super efficiency was given in [26]. The largest class of convex cones ensuring the existence of the efficient points in non-empty, compact subsets of the linear topological vector spaces was presented in [27], completed by the subsequent studies described in $[28,29]$ as was the last extension of the ordering convex cones to investigate the efficiency in Hausdorff locally convex spaces under completeness instead of compactness which is performed by Isac's cones; also used in the study of the geometrical aspects for Ekeland's principle [30]. Taking into account Corollary 3 of Theorem 1 in [3], we obtain:

Theorem 2.1 If $\left(X, P=\left\{p_{\alpha}: \alpha \in I\right\}\right)$ is a separated locally convex space with the topology generated by a family $P$ of seminorms and $A$ is a non-empty complete set in $X$ such that for every $p_{\alpha} \in P$ there exists a 
lower semicontinuous function $\varphi_{\alpha}: A \rightarrow R_{+} \quad$ with $\quad p_{\alpha}(x-y) \leq \varphi_{\alpha}(x)-\varphi_{\alpha}(y), \forall x \in A, y \in \Gamma(x), \quad$ then $\operatorname{MIN}_{K}(A) \neq \phi$.

The importance of Isac's cones concerning the existence and the domination property of the critical points sets for the corresponding dynamical systems is also illustrated by the following results.

Theorem 2.2 [18] Let $A \subseteq B \subseteq A+K$. If $K$ is an Isac's cone and $B \cap\left(A_{0}-K\right)$ is bounded and complete for some non-empty set $A_{0} \subseteq A$, then $\operatorname{MIN}_{K}(A) \neq \phi$.

Corollary 2.2.1 Let $A \subseteq B \subseteq A+K$. If $K$ is a weak Isac's cone and $B \cap\left(A_{0}-K\right)$ is bounded and weakly complete for some non-empty set $A_{0} \subseteq A$, then $\operatorname{MIN}_{K}(A) \neq \phi$.

In particular, if $K$ is a weak Isac's cone in $X$ and $A \cap(a-K)$ or $(A+K) \cap(a-K)$ is bounded and weakly complete for some $a \in A$, then $\operatorname{MIN}_{K}(A) \neq \phi$. When the boundedness and weak completeness properties hold for every $a \in A$, then we have the following domination property: $A \subseteq M I N_{K}(A)+K$.

Since in every separated locally convex space any normal cone is a weak Isac's cone (Proposition 2 of [14]), the conclusion of the above corollary remains valid whenever $K$ is a normal cone. Using this remark, one obtains the next existence results for the efficient points.

Corollary 2.2.2 $\operatorname{MIN}_{K}(A) \neq \phi$ if one of the following conditions holds:

(i) $K$ is closed, normal, weakly complete and $A$ is weakly closed such that $A \cap(a-K)$ is bounded for some $a \in A$. We also have $A \subseteq M I N_{K}(A)+K$ if, under the above hypotheses, $A \cap(a-K)$ is bounded for every $a \in A$,

(ii) $K$ is closed, normal and $A$ is bounded and weakly complete. The domination property holds again,

(iii) $K$ is closed, normal, weakly complete and $A+K$ is weakly closed such that $(A+K) \cap(a-K)$ is bounded for some $a \in A$.

The domination property $A \subseteq \operatorname{MIN}_{K}(A)+K$ holds if, in addition, $(A+K) \cap(a-K)$ is bounded for any $a \in A$.

Remark 2.2 Taking into account Corollary of Proposition 2 in [14] it is clear that all the above results remain valid if one replaces the hypothesis of normality on $K$ with the usual weak normality.

Corollary 2.2.3 If $A$ is a non-empty, bounded and closed subset of $X$ and $K$ is well based (that is, generated by a non-empty convex, bounded set which does not contain the origin of the space in its closure) by a complete set, then $\operatorname{MIN}_{K}(A) \neq \phi$ and $A \subseteq M I N_{K}(A)+K$.

Corollary 2.2.4 If $A$ is a non-empty, bounded and closed subset of a Banach space ordered by $a$ convex cone $K$ well based by a closed set, then $\operatorname{MIN}_{K}(A) \neq \phi$ and $A \subseteq M I N_{K}(A)+K$.

Remark 2.3 Since in a normed linear space a convex cone is an Isac's cone if it is well based, the above last theorem and its immediate corollaries offers useful conclusions for the efficiency whenever $K$ is an Isac's cone. Moreover, the existence results given in this section show also the possibility to use the (weakly) complete cones for the study of the existence and the properties of the solutions for the vector optimization problems in Hausdorff locally convex spaces, with appropriate numerical methods and they generalize similar conclusions to those indicated in [24]. 


\section{Coincidence Results between Equilibrium Point Sets and Choquet's Boundaries under Related Topics}

In our further considerations we suppose that $(E, \tau)$ is a Hausdorff locally convex space having its origin $\theta$ and $\tau$ its topology, $K$ is a closed, convex pointed cone in $E$ and $\varepsilon$ is an arbitrary element of $K \backslash\{\theta\}$. On the vector space $E$ we consider the usual order relation $\leq_{K}$ associated with $K$ as follows: for $x, y \in E$ one defines $x \leq_{K} y$, if $y \in x+K$.

Clearly, this order relation on $E$ is topologically closed, that is, the set $G_{K}$ given by $G_{K}=\left\{(x, y) \in E \times E: x \leq_{K} y\right\}$ is a closed subset of $E \times E$. Also, the set $G_{\varepsilon+K}=\{(x, y) \in E \times E: y \in x+\varepsilon+K\}$ is closed in $E \times E$ endowed with the usual product topology.

Definition 3.1 If $A$ is a non-empty subset of $E$, then $a_{0} \in A$ is called an $\varepsilon$-critical point (or, $\varepsilon$-minimal element, $\varepsilon$-efficient point, $\varepsilon$-near to minimum point) of $A$ with respect to $K$ if there exists no a $\in A$ such that $a_{0}-a-\varepsilon \in K$, that is, $A \cap\left(a_{0}-\varepsilon-K\right)=\phi$.

The $\varepsilon$-critical points set of $A$ with respect to $K$ will be denoted by $\varepsilon$ - $\operatorname{MIN}_{K}(A)$ or $\varepsilon$-eff $(A, K)$ following the analogy with [31-33].

Remark 3.1 It is clear that the concept of the $\varepsilon$-efficient element does not include the notion of the efficient point, but the immediate connection between them is represented by the relations $M I N_{K}(A) \subseteq \varepsilon-\operatorname{MIN}_{K}(A), \forall \varepsilon \in K \backslash\{\theta\}$ and $\operatorname{MIN}_{K}(A)=\bigcap_{\varepsilon \in K \backslash\{\theta\}}\left[\varepsilon-\operatorname{MIN}_{K}(A)\right]$.

Remark 3.2 A very interesting and important generalization of the approximate efficiency given by Definition 3.1 was considered in [33] by replacing $\varepsilon$ with a non-empty subset of $K \backslash\{\theta\}$. In this way, it was shown that the existence of this new type of efficient points for the lower bounded sets characterizes the semi-Archimedian ordered vector spaces and the regular ordered locally convex spaces. In [32] we also find many pertinent examples and comments inside the usual Euclidean spaces.

Definition 3.2 A real function $f: E \rightarrow \Re$ is called $\varepsilon+K$-increasing if $f\left(x_{1}\right) \geq f\left(x_{2}\right)$ whenever $x_{1}, x_{2} \in E$ and $x_{1} \in x_{2}+\varepsilon+K$.

For a non-empty and compact subset $X$ of $E$ we recall some basic considerations in Potential Theory concerning the Choquet boundary of $X$ with respect to a convex cone of continuous functions on $X$. Thus, we remember that if $S$ is a convex cone of real continuous functions on $X$ such that the constant function on $X$ belong to $S$, it is min-stable (i.e., for every $f_{1}, f_{2} \in S$ it follows $\inf \left(f_{1}, f_{2}\right) \in S$ ) and it separates the points of $X$, then on the set $M_{+}(X)$ of all positive Radon measures on $X$ one associates the following order relation:

If $\mu, v \in M_{+}(X)$ then $\mu \leq s v$, if $\mu(s) \leq v(s)$ for all $s \in S$.

Following [5] a measure $\mu \in M_{+}(X)$ is minimal with respect to the above order relation if for any continuous function $f: X \rightarrow \mathfrak{R}$ we have $\mu\left(Q_{s} f\right)=\mu(f)$, where $\mu\left(Q_{s} f\right)=\inf \{s \in S: f \leq s\}$. Particularly, if $x \in X$, then the Dirac measure $\varepsilon_{x}$ is minimal, if $\varepsilon_{x}\left(Q_{s} f\right)=\varepsilon_{x}(f)$, that is, $Q_{s} f(x)=f(x)$ for every continuous function $f: X \rightarrow \Re$.

The set of all points $x \in X$ such that $\varepsilon_{x}$ is minimal measure with respect to $\leq_{s}$ is named the Choquet boundary of $X$ with respect to $S$ and it is denoted by $\delta_{s} X$. Hence, if $C(X)$ is the usual Banach space of all real continuous functions on $X$, then 


$$
\delta_{s} X=\left\{x \in X: Q_{s} f(x)=f(x), \forall f \in C(X)\right\}
$$

A closed set $A \subseteq X$ is called $S$-absorbent if $x \in A$ and $\mu \leq_{s} \varepsilon_{x}$ implies that $\mu(X \backslash A)=0$. The trace on $\delta_{s} X$ of the topology on $X$ in which the closed sets coincide with $X$ or with the absorbent subsets of $X$ contained in such a set as $\{x \in X: \exists s \in S$ with $s(x)<\theta\}$ is usually named the Choquet topology on $\delta_{s} X$.

An important connection between the Vector Optimization and the Potential Theory is the next coincidence result on the efficient points sets and Choquet's boundaries in Hausdorff separated locally convex spaces, which cannot be obtained as a consequence of the Axiomatic Potential Theory, with significant details in [8-10,34], respectively.

Theorem 3.1 [8] $\operatorname{MIN}_{K}(X)$ coincides with the Choquet boundary of $X$ with respect to the convex cone of all real continuous functions which are increasing with respect to the order relation $\leq_{K}$. Consequently, the set $\operatorname{MIN}_{K}(X)$ endowed with the trace topology $\tau_{X}$ induced on $X$ by $\tau$ is a Baire space. Moreover, if $X$ is metrizable, then $\operatorname{MIN}_{K}(X)$ is a $G_{\delta}$ - set in $\left(X, \tau_{X}\right)$.

Taking into account Theorem 2.11 and Theorem 2.12 respectively in [5] we obtain

Corollary 3.1.1 (i) $\operatorname{MIN}_{K}(X)$ and $\operatorname{MIN}_{K}(X) \cap\{x \in X: s(x) \leq 0\}(s \in S)$ are compact sets with respect to Choquet's topology;(ii) $\operatorname{MIN}_{K}(X)$ is a compact subset of $X$.

Remark 3.3 In the conditions of Theorem 3.1, let us consider $M I N_{K}(X)$ endowed with the trace topology also denoted by $\tau_{X}$, the following game between partners $A$ and $B$. each partner successively chooses a non-empty set belonging to $\tau_{X}$ such that the player $A$ makes the first choice and each player must choose a set in $\tau_{X}$ which should be included in the previously chosen set of the other player.

Let $G_{1}, G_{1}^{\prime}, G_{2}, G_{2}^{\prime}, \ldots, G_{n}, G_{n}^{\prime}, \ldots$ be the successive options of the two players, $\left(G_{1}, G_{2}, \ldots, G_{n}, \ldots\right)$ represent the option expressed by $A$ and $\left(G_{1}^{\prime}, G_{2}^{\prime}, \ldots, G_{n}^{\prime}, \ldots\right)$ the option made by $B$. One says that player $B$ wins regardless of the way $A$ plays, he is able to make an option so that

$$
\bigcap_{x \in \square^{\infty}} G_{n} \neq \phi
$$

Theorem 3.1 together with Choquet's results (see, for examples, Chapter 2 of [5]) concerning the properties of the Choquet boundary shows that the above game on $\operatorname{MIN}_{K}(X)$ is won by player $B$.

Remark 3.4 As we have seen in [8] under the hypotheses of Theorem 3.1, the set eff $(X, K)$ coincides with the Choquet boundary of $X$ only with respect to the convex cone of all real, continuous and $K$-increasing functions on $X$. Thus, for example, if $X$ is a non-empty, compact and convex subset of $E$, then, taking into account the Theorem 2.2 in the first paragraph of Chapter 2 [5], the Choquet boundary of $X$ with respect to the convex cone of all real, continuous and concave functions on $X$ coincides with the set of all extreme points for $A$, that is, with the set of elements $x \in X$ such that if $x_{1}, x_{2} \in X, \lambda \in(0,1)$ and $x=\lambda x_{1}+(1-\lambda) x_{2}$, then $x=x_{1}=x_{2}$. But, it is clear that, even in infinite dimensional cases, an extreme point for a compact convex set is not necessarily an efficient point.

The following result extends Theorem 3.1 for $\varepsilon$-efficiency.

Theorem 3.2 [35] If $X$ is a non-empty subset of $E$, then the set $\varepsilon$-eff $(X, K)$ coincides with the Choquet boundary of $X$ with respect to the convex cone all $\varepsilon+K$-increasing real continuous functions on $X$. 
Consequently, the set $\varepsilon-e f f(X, K)$ endowed with the trace topology is a Baire space and if $\left(X, \tau_{X}\right)$ is metrizable, then $\varepsilon$-eff $(X, K)$ is a $G_{\delta}$-subset of $X$.

\section{A Generalized Modality for the Equilibrium Points Sets}

Let $X$ be a vector space with its origin denoted by $\theta$ ordered by a convex cone $K, K_{1}$ a non-void subset of $K$ and $A$ a non-empty subset of $X$. The following definition introduces a new concept of approximate efficiency in Hausdorff locally convex spaces which particularly leads to the well-known notion of Pareto type efficiency in the usual Euclidean spaces.

Definition 4.1 We say that $a_{0} \in A$ is a $K_{1}$-efficient (minimal) point of $A$, in notation, $a_{0} \in \operatorname{eff}\left(A, K, K_{1}\right)\left(\right.$ or $\left.a_{0} \in M I N_{K+K_{1}}(A)\right)$ if it satisfies one of the following equivalent conditions:

(i) $A \cap\left(a_{0}-K-K_{1}\right) \subseteq a_{0}+K+K_{1}$

(ii) $\left(K+K_{1}\right) \cap\left(a_{0}-A\right) \subseteq-K-K_{1}$

In a similar manner one defines the maximal efficient points by replacing $K+K_{1}$ with $-\left(K+K_{1}\right)$.

Remark 4.1 $a_{0} \in \operatorname{eff}\left(A, K, K_{1}\right)$, if it is a fixed point for the multifunction $F: A \rightarrow A$ defined by $F(t)=\left\{a \in A: A \cap\left(a-K-K_{1}\right) \subseteq t+K+K_{1}\right\}$.

Remark 4.2 In [33] it was shown that whenever $K_{1} \subset K \backslash\{\theta\}$, the existence of this new type of efficient points for the lower bounded sets characterizes the semi-Archimedian ordered vector spaces and the regular ordered locally convex spaces.

Remark 4.3 When $K$ is pointed, then $a_{0} \in \operatorname{eff}\left(A, K, K_{1}\right)$ means that $A \cap\left(a_{0}-K-K_{1}\right)=\varnothing$ or, equivalently, $\left(K+K_{1}\right) \cap\left(a_{0}-A\right)=\varnothing$ for $\theta \notin K_{1}$ and $A \cap\left(a_{0}-K-K_{1}\right)=\left\{a_{0}\right\}$, respectively, if $\theta \in K_{1}$. Whenever $K$ is pointed and $K_{1}=\{\theta\}$, from Definition 4.1, one obtains the well-known notion of the efficient (minimal, optimal or admissible) point, abbreviated $a_{0} \in \operatorname{eff}(A, K)\left(a_{0} \in M I N_{K}(A)\right)$, that is, satisfying the next equivalent properties:

(i) $A \cap\left(a_{0}-K\right)=\left\{a_{0}\right\}$

(ii) $A \cap\left(a_{0}-K \backslash\{\theta\}\right)=\varnothing$

(iii) $K \cap\left(a_{0}-A\right)=\{\theta\}$

(iv) $(K \backslash\{\theta\}) \cap\left(a_{0}-A\right)=\varnothing$

and we notice that

$$
\text { eff }\left(A, K, K_{1}\right)=\operatorname{eff}(A, K)=\bigcap_{\{\theta\} \neq K_{2} \subseteq K} \operatorname{eff}\left(A, K, K_{2}\right)
$$

It is clear that for any $\varepsilon \in K \backslash\{\theta\}$, taking $K_{1}=\{\varepsilon\}$, it follows that $a_{0} \in e f f\left(A, K, K_{1}\right)$ if, and only if, $A \cap\left(a_{0}-\varepsilon-K\right)=\varnothing$. In all these cases, the set eff $\left(A, K, K_{1}\right)$ was denoted by $\varepsilon-e f f(A, K)\left(\right.$ or $\varepsilon-M I N_{K}(A)$ as in [11]) and it is obvious that eff $(A, K)=\bigcap_{\varepsilon \in K \backslash\left\{\theta_{\}}\right.}[\varepsilon-e f f(A, K)]$.

Remark 4.3 The following theorem offers the first important connection between the strong optimization and the approximate efficiency in the environment of the ordered vector spaces, described initially in the previous Definition 4.1. 
Theorem 4.1 If we denote by $S\left(A, K, K_{1}\right)=\left\{a_{1} \in A: A \subseteq a_{1}+K+K_{1}\right\}$ and $S\left(A, K, K_{1}\right) \neq \varnothing$, then $S\left(A, K, K_{1}\right)=e f f\left(A, K, K_{1}\right)$.

Proof Clearly, $S\left(A, K, K_{1}\right) \subseteq e f f\left(A, K, K_{1}\right)$. Indeed, if $a_{0} \in S\left(A, K, K_{1}\right)$ and $a \in A \cap\left(a_{0}-K-K_{1}\right)$ are arbitrary elements, then $a \in a_{0}+K+K_{1}$, that is, $a_{0} \in \operatorname{eff}\left(A, K, K_{1}\right)$, by virtue of (i) in Definition 4.1. Suppose now that $\bar{a} \in S\left(A, K, K_{1}\right) \neq \varnothing$ and there exists $a_{0} \in e f f\left(A, K, K_{1}\right) \backslash S\left(A, K, K_{1}\right)$. From $\bar{a} \in S\left(A, K, K_{1}\right)$ it follows that $a_{0} \in \bar{a}+K+K_{1}$, that is, $\bar{a} \in a_{0}-K-K_{1}$, from which, since $\bar{a} \in A$ and $a_{0} \in \operatorname{eff}\left(A, K, K_{1}\right)$ we conclude that $\bar{a} \in a_{0}+K+K_{1}$. Therefore, $A \subseteq \bar{a}+K+K_{1} \subseteq a_{0}+K+K_{1}$, in contradiction with $a_{0} \notin S\left(A, K, K_{1}\right)$ as claimed.

Remark 4.4 If $S\left(A, K, K_{1}\right) \neq \varnothing$, then $K+K_{1}=K$ hence eff $\left(A, K, K_{1}\right)=$ eff $(A, K)$. Indeed, let $a \in S\left(A, K, K_{1}\right)$. Then, $a \in a+K+K_{1}$ which implies that $0 \in K+K_{1}$.

Therefore, $K \subseteq K_{1}+K+K=K_{1}+K \subseteq K$. The above theorem shows that, for any non-empty subset of an arbitrary vector space, the set of all strong minimal elements with respect to any convex cone, through the agency of every non-void subset of it, coincides with the corresponding set of the efficient (minimal) points whenever there is at least a strong minimal element, the result remaining obviously valid for the strong maximal elements and the maximal efficient points, respectively. Using this result and our abstract construction given in [36] for the H-locally convex spaces introduced by Th. Precupanu in [37], as separated locally convex spaces with any seminorms satisfying the parallelogram law, we established in [16] that the only simultaneous and vectorial approximation for each element, in the direct sum of a (closed) linear subspace and its orthogonal with respect to a linear (continuous) operator between two H-locally convex spaces, is its spline function. We also note that it is possible to have $S\left(A, K, K_{1}\right)=\varnothing$ and $\operatorname{eff}\left(A, K, K_{1}\right)=A$. Thus, for example, if one considers $X=R^{n}(n \in N, n \geq 2)$ endowed with the separated H-locally convex topology generated by the semi-norms $p_{i}: X \rightarrow R_{+}, p_{i}(x)=\left|x_{i}\right|, \forall x=\left(x_{i}\right) \in X, i=\overline{1, n}, K=R_{+}^{n}, K_{1}=\{(0, \ldots, 0)\}$ and for each real number $c$ we define $A_{c}=\left\{\left(x_{i}\right) \in X: \sum_{i=1}^{n} x_{i}=c\right\}$, then it is clear that $S\left(A_{c}, K, K_{1}\right)$ is empty and eff $\left(A_{c}, K, K_{1}\right)=A_{c}$.

In all our further considerations we suppose that $X$ is a Hausdorff locally convex space having the topology induced by family $P=\left\{p_{\alpha}: \alpha \in I\right\}$ of seminorms, ordered by a convex cone $K$ and its topological dual space $X^{*}$. In this framework, the next theorem contains a significant criterion for the existence of the approximate Pareto (minimal) efficient points, in particular, for the usual Pareto (minimal) efficient points, taking into account that the dual cone of $K$ is defined by $K^{*}=\left\{x^{*} \in X^{*}: x^{*}(x) \geq 0, \forall x \in K\right\}$ and its attached polar cone is $K^{0}=-K^{*}$. The version for the approximate maximal efficient points is straightforward.

Theorem 4.2 If $A$ is any non-empty subset of $X$, and $K_{1}$ is every non-void subset of $K$, then $a_{0} \in \operatorname{eff}\left(A, K, K_{1}\right)$ whenever for each $p_{\alpha} \in P$ and $\eta \in(0,1)$ there exists $x^{*}$ in the polar cone $K^{0}$ of $K$ such that $p_{\alpha}\left(a_{0}-a\right) \leq x^{*}\left(a_{0}-a\right)+\eta, \forall a \in A$. 
Proof Let us suppose that, under the above hypotheses, $\left(K+K_{1}\right) \cap\left(a_{0}-A\right) \nsubseteq \subseteq-\left(K+K_{1}\right)$, that is, there exists $a \in A$ so that $a_{0}-a \in K+K_{1} \backslash\left(-K-K_{1}\right)$. Then, $a_{0}-a \neq 0$ and, because $X$ is separated in Hausdorff's sense, there exists $p_{\alpha} \in P$ such that $p_{\alpha}\left(a_{0}-a\right)>0$. On the other hand, there exists $n \in N^{*}$ sufficiently large with $p_{\alpha}\left(a_{0}-a\right) / n \in(0,1)$ and the relation given by the hypothesis of theorem leads to $p_{\alpha}\left(a_{0}-a\right) \leq x^{*}\left(a_{0}-a\right)+p_{\alpha}\left(a_{0}-a\right) / n$ with $x^{*} \in K^{0}$ and $n \rightarrow \infty$, which implies that $p_{\alpha}\left(a_{0}-a\right) \leq 0$, is a contradiction and the proof is complete.

Remark 4.5 The above theorem represents an immediate extension of Precupanu's result given in Proposition 1.2 of [38]. In general, the converse of this theorem is not valid at least in (partially) ordered separated locally convex spaces as we can see from the example considered in Remark 4.4. Indeed, if one assumes the contrary in the corresponding, mathematical calculation's background, then, taking $\eta=\frac{1}{4} \quad$ it follows that for each $\lambda_{0} \in[0,1]$ there exists $c_{1}, c_{2} \leq 0$ such that $\left|\lambda_{0}-\lambda\right| \leq\left(c_{1}-c_{2}\right)\left(\lambda_{0}-\lambda\right)+\frac{1}{4}, \forall \lambda \in[0,1] . \quad$ Taking $\quad \lambda_{0}=\frac{1}{4} \quad$ one obtains $\quad|1-4 \lambda| \leq\left(c_{1}-c_{2}\right)(1-4 \lambda)+1, \forall \lambda \in[0,1]$ which for $\lambda=0$ implies that $c_{2} \leq c_{1}$ and for $\lambda=\frac{1}{2}$ leads to $c_{1} \leq c_{2}$, that is, $|1-4 \lambda| \leq 1, \forall \lambda \in[0,1]$, a contradiction.

The content of Section 4 in [17] motivated us to consider for each function $\varphi: P \rightarrow K^{*} \backslash\{0\}$ the full nuclear cone $K_{\varphi}=\left\{x \in X: p_{\alpha}(x) \leq \varphi\left(p_{\alpha}\right)(x), \forall p_{\alpha} \in P\right\}$ and to give the next generalization of Theorem 7 [17] in a more general context, which represents also a new important link between strong optimization and the approximate vector optimization together with its usual particular variant, respectively.

Theorem 4.3 If there exists $\varphi: P \rightarrow K^{*} \backslash\{0\}$, then,

$$
\operatorname{eff}\left(A, K, K_{1}\right)=\bigcup_{\substack{a \in A \\ \varphi \in P \rightarrow K^{*} \backslash\{0\}}} S\left(A \cap\left(a-K-K_{1}\right), K_{\varphi}\right)
$$

for any non-empty subset $K_{1}$ of $K$.

Proof If $a_{0} \in e f f\left(A, K, K_{1}\right)$ is an arbitrary element, then, in accordance with point (i) of the Definition 4.1 and the hypothesis of the above theorem, we have $A \cap\left(a_{0}-K-K_{1}\right)-a_{0} \subseteq K+K_{1} \subseteq K \subseteq K_{\varphi}$ for some $\varphi: P \rightarrow K^{*} \backslash\{0\}$.

Therefore, $a_{0} \in S\left(A \cap\left(a_{0}-K-K_{1}\right), K_{\varphi}\right)$.

Hence, eff $\left(A, K, K_{1}\right) \subseteq \bigcup_{\substack{a \in A \\ \varphi: P \rightarrow K^{*} \backslash\{0\}}} S\left(A \cap\left(a_{0}-K-K_{1}\right), K_{\varphi}\right)$. Conversely, consider now $a_{1} \in S\left(A \cap\left(a_{0}-K-K_{1}\right), K_{\varphi}\right)$ for $a_{0} \in A$ and $\varphi: P \rightarrow K^{*} \backslash\{0\}$. Then, $a_{1} \in A \cap\left(a_{0}-K-K_{1}\right)$ and $A \cap\left(a_{0}-K-K_{1}\right)-a_{1} \subseteq K_{\varphi}$, that is,

$p_{\alpha}\left(a-a_{1}\right) \leq \varphi\left(p_{\alpha}\right)\left(a-a_{1}\right), \forall a \in A \cap\left(a_{0}-K-K_{1}\right), p_{\alpha} \in P$ which implies immediately that $p_{\alpha}\left(a_{1}-a\right) \leq-\varphi\left(p_{\alpha}\right)\left(a_{1}-a\right)+\eta, \forall a \in A \cap\left(a_{0}-K-K_{1}\right), \quad p_{\alpha} \in P, \eta \in(0,1)$ and, by virtue of Theorem 4.2 one obtains that $a_{1} \in e f f\left(A \cap\left(a_{0}-K-K_{1}\right), K, K_{1}\right)$.

But eff $\left(A \cap\left(a_{0}-K-K_{1}\right), K, K_{1}\right) \subseteq e f f\left(A, K, K_{1}\right)$. 
Indeed, for any $t \in e f f\left(A \cap\left(a_{0}-K-K_{1}\right), K, K_{1}\right) \quad$ and $h \in A \cap\left(t-K-K_{1}\right) \quad$ we have $h \in A \cap\left(a_{0}-K-K_{1}\right) \cap\left(t-K-K_{1}\right) \subseteq t+K+K_{1}$, that is, $A \cap\left(t-K-K_{1}\right) \subseteq t+K+K_{1}$ and by the point (i) of Definition 4.1 it follows that $t \in e f f\left(A, K, K_{1}\right)$. This completes the proof.

Remark 4.6 The hypothesis $K \subseteq K_{\varphi}$ imposed upon the convex cone $K$ is automatically satisfied whenever $K$ is an Isac's cone and it was used only to prove the inclusion eff $\left(A, K, K_{1}\right) \subseteq \bigcup_{\substack{a \in A \\ \varphi: P \rightarrow K^{\vee}\{0\}}} S\left(A \cap\left(a-K-K_{1}\right), K_{\varphi}\right)$. When $K$ is any pointed convex cone, $A$ is a non-empty subset of $X$ and $a_{0} \in e f f(A, K)$, then, by virtue of (i) in Remark 4.3, it follows that $A \cap\left(a_{0}-K\right)=\left\{a_{0}\right\}$, that is, $A \cap\left(a_{0}-K\right)-a_{0}=\{0\} \subset K_{\varphi}$. Hence, $a_{0} \in S\left(A \cap\left(a_{0}-K\right), K_{\varphi}\right)$ for every mapping $\varphi: P: K^{*} \backslash\{0\}$ and the next corollary is valid.

Corollary 4.3.1 For every non-empty subset A of any Hausdorff locally convex space ordered by an arbitrary, pointed convex cone $K$ with its dual cone $K^{*}$ we have

$$
\operatorname{eff}(A, K)=\bigcup_{\substack{a \in A \\ \varphi: P \rightarrow K^{*} \backslash\{0\}}} S\left(A \cap(a-K), K_{\varphi}\right)
$$

Remark 4.7 Clearly, the announced theorem represents a significant result concerning the possibilities of scalarization for the study of the above efficiency in separated locally convex spaces, so for the particular cases of Hausdorff locally convex spaces ordered by closed, pointed and normal cones. The coincidence between the equilibrium points sets and Choquet's boundaries together with its immediate corollaries remains valid if one replaces $\varepsilon$ by $K_{1}$.

\section{Conclusions}

The main goal of this paper was to exhibit some pertinent results on the equilibrium point sets for the generalized dynamical systems with respect to Isac's cones. The relations between this kind of equilibria, the efficiency in the infinite dimensional ordered vector spaces and Choquet's boundaries were also examined.

\section{Acknowledgements}

The author would like to thank the anonymous referees for their valuable suggestions and conclusions concerning this work.

\section{References and Notes}

1. Peressini, A.L. Ordered Topological Vector Spaces; Harper and Row: New York, NY, USA, 1967.

2. Isac, G. Points Critiques Pour Des Systèmes Dynamiques. In Cônes Nucleaires et Optimum de Pareto; Royal Military College of St. Jean: Québec, Canada, 1981.

3. Isac, G. Sur l'existence de l'optimum de Pareto. Rivista di Matematica della Università di Parma 1983, 4, 303-325. 
4. Becker, R. Sur les cones faiblement comples contenus dans le dual d'un space de Banach nonreflexif. Bull. Aust. Math. Soc. 1989, 39, 321-328.

5. Boboc, N.; Bucur, G.H. Convex Cones of Continuous Functions on Compact Spaces; Publishing House of the Roumanian Academy: Bucharest, Romania, 1976.

6. Bucur, G.H. Couples normaux de quasi-topologies. Théorèmes de prolongement. Revue Roumaine de Mathématiques Pures et Appliquées 1969, 14, 1395-1422.

7. Bucur, G.H. Cônes convexes des fonctions continues sur un espace compact. Revue Roumaine de Mathématiques Pures et Appliquées 1971, 14, 1441-1460.

8. Bucur, I.; Postolică, V. A coincidence results between, sets of efficient points and Choquet boundaries is separated locally convex spaces. Optimization 1996, 36, 231-234.

9. Choquet, G. Lecture on Analysis 1-3. In Mathematics Lecture Notes Series; Benjamin Publishing House: Benjamin, New York, Amsterdam, 1969.

10. Constantinescu, C. L'ensemble des function surharmoniques positive sur un espace harmonique est faiblement complet. Comptes Rendus de l'Académie des Sciences 1970, 271, A549-A551.

11. Postolică, V. Approximate efficiency in infinite dimensional ordered vector spaces. Int. J. Appl. Manag. Sci. 2009, 1, 300-314.

12. Bahya, A.O. Ensembles coniquement bornés et cônes nucléaires dans les éspaces localement convexes separés. Ph.D. Thesis, L’École Normale Superieure, Takaddoum Rabat, Maroc, 1989.

13. Bahya, A.O. Etudes des cones nucleaires. Annales des Science Mathématique du Québec 1992, $15,123-134$.

14. Isac, G. Supernormal cones and absolute summability. Libertas Mathematica 1985, 5, 17-32.

15. Isac, G. Pareto optmization in infinite dimensional spaces: the importance of nuclear cones. $J$. Math. Anal. Appl. 1994, 182, 393-404.

16. Isac, G.; Postolică, V. The Best Approximation and Optimization in Locally Convex Spaces; Verlag Peter Lang Publishing House: Frankfurt am Main, Germany, 1993.

17. Isac, G.; Bahya, A.O. Full nuclear cones associated to a normal cone. Application to Pareto efficiency. Appl. Math. Lett. 2002, 15, 633-639.

18. Postolică, V. New existence results for efficient points in locally convex spaces ordered by supernormal cones. J. Glob. Optim. 1993, 3, 233-242.

19. Postolică, V. An extension to sets of supernormal cones and generalized subdifferentials. Optimization 1994, 29, 131-139.

20. Postolică, V. Properties of Pareto sets in locally convex spaces. Optimization 1995, 34, 223-229.

21. Pietch, A. Nuclear Locally Convex Spaces; Springer-Verlag: Berlin, Germany, 1972.

22. Krasnoselski, M.A. Positive Solutions of Operator Equations; Noordhoff Publishing House: Groningen, Noorhoff, 1964.

23. Nachbin, L. Topology and Order; Van Nostrand: New York, NY, USA, 1965.

24. Luc, D.T. Theory of Vector Optimization; Springer-Verlag: Berlin, Germany, 1989.

25. Staib, T. On two generalizations of Pareto minimality. J. Optim. Theory Appl. 1988, 59, 289-306.

26. Borwein, J.M.; Zhuang, D. Super efficiency in vector optimization. Trans. Am. Math. Soc. 1993, $338,105-122$.

27. Karwat, A. Sterna, on the existence of cone maximal points in real topological linear spaces. Israel J. Math. 1986, 54, 33-41. 
28. Ha, T.X.D. On the existence of efficient points in locally convex spaces. J. Global Optim. 1994, 4, 265-278.

29. Ha, T.X.D. A note on a class of cones ensuring the existence of efficient points is bounded complete sets. Optimization 1994, 31, 141-145.

30. Tammer, C. A generalization of Ekeland's variational principle. Optimization 1992, 25, 129-141.

31. Loridan, P. $\varepsilon$-solutions of vector minimization problems. J. Optim. Theory Appl. 1984, 43, 65-276.

32. Nemeth, A.B. Near to minimality in ordered vector spaces. Mathematica 1981, 23, 239-243.

33. Nemeth, A.B. Between Pareto efficiency and Pareto $\varepsilon$-efficiency. Optimization 1989, 20, 615-637.

34. Mokobodzki, G. Principe de balayage, principe de domination, Paris. Seminaire Choquet Initiation à l'analyse 1962, 1, 1-11.

35. Postolică, V. Calculus of Choquet boundaries using Pareto sets. In Lecture Notes in Economics and Mathematical Systems 448; Springer-Verlag: Berlin, Germany, 1997; pp. 47-54.

36. Postolică, V. Spline functions in H-locally convex spaces. Annales Scientifiques de l'Université" Al.I.Cuza." de Jassy 1981, 27, 333-338.

37. Precupanu, T. Espaces linéaires à semi-norms Hilbertiennes. Annales Scientifiques de l'Université"Al.I.Cuza." de Jassy 1969, 115, 83-93.

38. Precupanu, T. Scalar minimax properties in vectorial optimization. Numer. Math. 1992, 107, 299-306.

(C) 2012 by the authors; licensee MDPI, Basel, Switzerland. This article is an open access article distributed under the terms and conditions of the Creative Commons Attribution license (http://creativecommons.org/licenses/by/3.0/). 